\title{
Efficient Solution of the Electric-Field Integral Equation Using the Iterative LSQR Algorithm
}

\author{
Özgür Ergül, Student Member, IEEE, and Levent Gürel, Senior Member, IEEE
}

\begin{abstract}
In this letter, we consider iterative solutions of the three-dimensional electromagnetic scattering problems formulated by surface integral equations. We show that solutions of the electric-field integral equation (EFIE) can be improved by employing an iterative least-squares QR (LSQR) algorithm. Compared to many other Krylov subspace methods, LSQR provides faster convergence and it becomes an alternative choice to the time-efficient no-restart generalized minimal residual (GMRES) algorithm that requires large amounts of memory. Improvements obtained with the LSQR algorithm become significant for the solution of large-scale problems involving open surfaces that must be formulated using EFIE, which leads to matrix equations that are usually difficult to solve iteratively, even when the matrix-vector multiplications are accelerated via the multilevel fast multipole algorithm.
\end{abstract}

Index Terms-Iterative algorithms, least-squares $\mathrm{QR}$ algorithm, multilevel fast multipole algorithm, scattering problems.

\section{INTRODUCTION}

$\mathbf{F}$ OR surface formulations of electromagnetic scattering problems, various integral equations can be employed either at our discretion, or due to limitations imposed by the problem. For solutions of problems with closed geometries, the combined-field integral equation (CFIE) is preferred over the electric-field integral equation (EFIE) and the magnetic-field integral equation (MFIE). This is because CFIE is free of the internal-resonance problem and it provides better-conditioned matrix equations [1], which are easier to solve iteratively compared to the matrix equations obtained with EFIE and MFIE. However, MFIE and CFIE are applicable only to closed geometries, leaving EFIE as the only choice for the solutions of various real-life problems of electromagnetics involving geometries modelled by open surfaces. For example, scattering problems involving a sphere geometry depicted in Fig. 1(a) can be formulated by EFIE, MFIE, and CFIE. However, only EFIE can be employed for the solution of problems involving a patch geometry shown in Fig. 1(b). Unfortunately, EFIE usually produces matrix equations that are difficult to solve iteratively [2].

Manuscript received March 2, 2007; revised September 5, 2007. This work was supported by the Scientific and Technical Research Council of Turkey (TUBITAK) under Research Grant 105E172, by the Turkish Academy of Sciences in the framework of the Young Scientist Award Program (LG/TUBA-GEBIP/2002-1-12), and by contracts from ASELSAN and SSM.

The authors are with the Department of Electrical and Electronics Engineering and Computational Electromagnetics Research Center (BiLCEM), Bilkent University, TR-06800, Bilkent, Ankara, Turkey (e-mail: ergul@ee.bilkent.edu.tr; lgurel@bilkent.edu.tr).

Color versions of one or more figures in this letter are available online at http://ieeexplore.ieee.org.

Digital Object Identifier 10.1109/LAWP.2007.908008

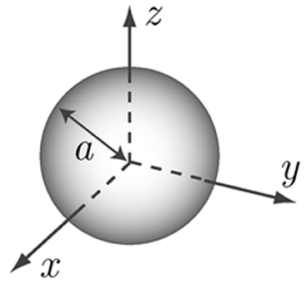

(a)

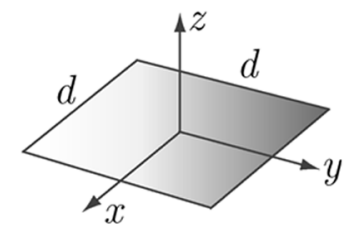

(b)
Fig. 1. Examples of closed and open geometries. (a) Sphere of radius $a$ (closed geometry) and (b) $d \times d$ square patch (open geometry).

There are many efforts to improve the convergence of the EFIE solutions by employing preconditioners [3]. Although different kinds of simple preconditioners, such as block-diagonal preconditioners, easily improve the convergence of the CFIE solutions, they usually do not reduce the number of iterations for EFIE [2]. As the problem size gets larger and the dimensions of the matrix equation grow, solutions with EFIE become extremely difficult, even when iterative techniques are used with acceleration methods, such as the multilevel fast multipole algorithm (MLFMA) [4] for the matrix-vector multiplications. Therefore, strong preconditioners are implemented to increase the convergence rates without increasing the computational complexity. In this way, it becomes possible to solve problems with millions of unknowns on relatively inexpensive clusters of personal computers, even when the geometries are open and the problems are formulated by the ill-conditioned EFIE [3].

In an iterative solution, the number of iterations naturally depends on the type of the iterative solver. Among the different kinds of algorithms, Krylov subspace methods, such as conjugate gradient (CG), conjugate gradient squared (CGS), biconjugate gradient (BiCG), stabilized BiCG (BiCGSTAB), quasiminimal residual (QMR), transpose-free QMR (TFQMR), and generalized minimal residual (GMRES) [5], can be employed for the solutions of various problems in computational electromagnetics. For a given problem, the performance of each algorithm may vary significantly, depending on the shape of the geometry, discretization, and the type of the formulation. Since reducing the number of iterations is extremely important to obtain efficient solutions, we investigate and compare the iterative solutions provided by various algorithms. In this letter, we show that a least-squares QR (LSQR) [6] algorithm provides improved convergence rates compared to other algorithms for the solutions of the problems formulated with EFIE. We also show that the improved convergence is peculiar to EFIE and LSQR does not have a good performance for CFIE solutions. Iterative 
solutions of relatively large problems involving a patch geometry are presented to compare the performance of LSQR with other algorithms that are commonly used in the literature.

\section{ITERATIVE LEAST-SQUARES SOLUTIONS OF INTEGRAL EQUATIONS}

For conducting surfaces, EFIE and MFIE can be written directly from the boundary conditions for the tangential electric and magnetic fields (in phasor notation with the $e^{-i w t}$ convention) as

$$
\hat{\boldsymbol{t}} \cdot \int_{S} d \boldsymbol{r}^{\prime} \boldsymbol{J}\left(\boldsymbol{r}^{\prime}\right) \cdot\left(\overline{\mathbf{I}}-\frac{\nabla \nabla^{\prime}}{k^{2}}\right) g\left(\boldsymbol{r}, \boldsymbol{r}^{\prime}\right)=\frac{i}{k \eta} \hat{\boldsymbol{t}} \cdot \boldsymbol{E}^{i}(\boldsymbol{r})
$$

and

$$
\boldsymbol{J}(\boldsymbol{r})-\hat{\boldsymbol{n}} \times \int_{S} d \boldsymbol{r}^{\prime} \boldsymbol{J}\left(\boldsymbol{r}^{\prime}\right) \times \nabla^{\prime} g\left(\boldsymbol{r}, \boldsymbol{r}^{\prime}\right)=\hat{\boldsymbol{n}} \times \boldsymbol{H}^{i}(\boldsymbol{r}),
$$

respectively. In (1) and (2), $\hat{\boldsymbol{t}}$ and $\hat{\boldsymbol{n}}$ are the tangential and outward normal unit vectors on the surface at the observation point $\boldsymbol{r}, \boldsymbol{E}^{\boldsymbol{i}}$ and $\boldsymbol{H}^{\boldsymbol{i}}$ are the incident electric and magnetic fields, $\boldsymbol{J}$ is the electric current induced on the surface, $k$ is the wavenumber, $\eta$ is the impedance of free space, and

$$
g\left(\boldsymbol{r}, \boldsymbol{r}^{\prime}\right)=\frac{e^{i k R}}{4 \pi R} \quad\left(R=\left|\boldsymbol{r}-\boldsymbol{r}^{\prime}\right|\right)
$$

denotes the free-space Green's function. Then, CFIE is defined as the convex combination of EFIE and MFIE as

$$
\mathrm{CFIE}=\alpha \mathrm{EFIE}+(1-\alpha) \frac{i}{k} \mathrm{MFIE}
$$

where $\alpha$ is a parameter between 0 and 1 .

By the simultaneous discretization of both the geometry and the integral equation applied to formulate the problem, we obtain an $N \times N$ matrix equation, i.e.

$$
\bar{Z} \cdot \boldsymbol{a}=\boldsymbol{v}
$$

where $\boldsymbol{a}$ represents the unknown coefficient vector, $\bar{Z}$ is the impedance matrix, and $\boldsymbol{v}$ is the excitation vector. Matrix elements are derived as

$$
\begin{aligned}
Z_{m n}^{E} & =\int_{S_{m}} d \boldsymbol{r} \boldsymbol{t}_{m}(\boldsymbol{r}) \cdot \int_{S_{n}} d \boldsymbol{r}^{\prime} g\left(\boldsymbol{r}, \boldsymbol{r}^{\prime}\right) \boldsymbol{b}_{n}\left(\boldsymbol{r}^{\prime}\right) \\
& -\frac{1}{k^{2}} \int_{S_{m}} d \boldsymbol{r}\left[\nabla \cdot \boldsymbol{t}_{m}(\boldsymbol{r})\right] \int_{S_{n}} d \boldsymbol{r}^{\prime} g\left(\boldsymbol{r}, \boldsymbol{r}^{\prime}\right)\left[\nabla^{\prime} \cdot \boldsymbol{b}_{n}\left(\boldsymbol{r}^{\prime}\right)\right]
\end{aligned}
$$

for EFIE and

$$
\begin{aligned}
Z_{m n}^{M}= & \int_{S_{m}} d \boldsymbol{r} t_{m}(\boldsymbol{r}) \cdot \boldsymbol{b}_{n}(\boldsymbol{r}) \\
& -\int_{S_{m}} d \boldsymbol{r} \boldsymbol{t}_{m}(\boldsymbol{r}) \cdot \hat{\boldsymbol{n}} \times \int_{S_{n}} d \boldsymbol{r}^{\prime} \boldsymbol{b}_{n}\left(\boldsymbol{r}^{\prime}\right) \times \nabla^{\prime} g\left(\boldsymbol{r}, \boldsymbol{r}^{\prime}\right)
\end{aligned}
$$

for MFIE, respectively, where $m, n=1,2, \ldots, N$. In (6) and (7), $\boldsymbol{b}_{n}$ and $\boldsymbol{t}_{m}$ represent the set of basis and testing functions with the spatial supports of $S_{n}$ and $S_{m}$, respectively.

For the least-squares solution of the matrix equation in (5), we perform the transformation

$$
\bar{Z} \cdot a=v \quad \rightarrow \quad \bar{Z}^{H} \cdot \bar{Z} \cdot a=\bar{Z}^{H} \cdot v
$$

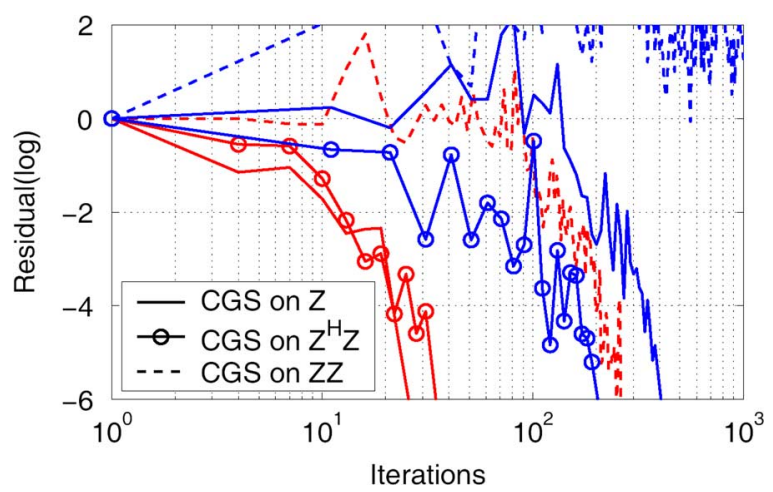

Fig. 2. Iterative solutions of a scattering problem involving a sphere of radius $1.5 \lambda$ formulated by CFIE (red) and EFIE (blue). Matrix equations have 8364 unknowns and the solutions are performed by the CGS algorithm applied on the ordinary equation and the transformed equations in (8) and (10), labeled by "Z ${ }^{H} \mathrm{Z}$ " and "ZZ", respectively.

with

$$
\bar{Z}^{\boldsymbol{H}}=\left\{\bar{Z}^{\boldsymbol{T}}\right\}^{*},
$$

where " $T$ " and "*”" represent the transpose and complex conjugate operations, respectively. We note that $\bar{Z}^{H} \cdot \bar{Z}$ is a Hermitian matrix. In this letter, we choose the basis and testing functions as Rao-Wilton-Glisson (RWG) [7] functions defined on planar triangles and apply the Galerkin method. Then, in the case of EFIE, the impedance matrix with the elements in (6) becomes symmetric and only the complex conjugate operation is required in (9). When the transformed matrix equation in (8) is solved iteratively using MLFMA, it is relatively easy to obtain the multiplications related to the complex conjugate matrix using the ordinary MLFMA with simple modifications. On the other hand, for the least-squares solution of a matrix equation involving a nonsymmetric impedance matrix, a transpose MLFMA should be implemented carefully by reversing the steps of the ordinary MLFMA. With the Galerkin method, least-squares solutions of MFIE with the matrix elements in (7), and therefore also CFIE, require the implementation of the transpose MLFMA.

Fig. 2 presents the iterative solutions of a scattering problem related to a sphere of radius $1.5 \lambda$, where the residual error is plotted with respect to the iteration count. The sphere is illuminated by a plane wave and the matrix equations with 8364 unknowns are solved by the CGS algorithm using MLFMA. For both the CFIE and the EFIE formulations, we compare the rate of convergence of the ordinary matrix equation in (5) and the transformed equation in (8). In addition, we also consider the transformation in the form of

$$
\bar{Z} \cdot a=v \quad \rightarrow \quad \bar{Z} \cdot \bar{Z} \cdot a=\bar{Z} \cdot v,
$$

where both sides of the ordinary matrix equation in (5) are multiplied with the impedance matrix.

We note that the new matrices in (8) and (10) obtained by the transformations, i.e., $\bar{Z}^{H} \cdot \bar{Z}$ and $\bar{Z} \cdot \bar{Z}$, have condition numbers that are the squares of the condition numbers of the impedance matrix $\bar{Z}$. With higher condition numbers, both transformations might decrease the convergence rates. However, Fig. 2 shows that this is not true and we obtain different results from the two transformations, especially for EFIE. 
1) The transformation in (10) decreases the convergence rates significantly for both CFIE and EFIE compared to the ordinary solutions. The transformed matrix equations require more iterations, especially in the case of EFIE, where convergence to an error of less than 0.1 cannot be achieved within 1000 iterations.

2) With the transformation in (8), CFIE has a faster convergence compared to the solution obtained by the transformation in (10), but it is still slower than the ordinary solution. However, in the case of EFIE, the transformation in (8) leads to an even faster convergence than the ordinary solution.

We also confirmed the results presented in Fig. 2 by considering various scattering problems involving different geometries with closed and open surfaces. We observed that the transformation in (8) consistently improved the convergence rate of the iterative solutions of the EFIE formulations.

It is shown in [8], [9] that a transformation similar to (10) improves the conditioning of EFIE and provides faster convergence in the iterative solutions, but not in the discrete form as in (10), and subject to a stabilization procedure that is valid only for closed surfaces. In this work, we show that the iterative solutions of EFIE can be improved by the transformation in (8) using the transpose complex conjugate of the impedance matrix. This improvement is obtained in the discrete form of the transformation as in (8), and it also exists for the solutions of problems involving open surfaces.

In Fig. 2, the transformed equation in (8) offers a faster convergence rate compared to the ordinary equation for EFIE. However, it does not increase the efficiency of the iterative solution since the transformed equation requires four matrix-vector products per CGS iteration while the ordinary solution requires only two. As a consequence, we suggest solving the transformed equation in (8) using the $\mathrm{CG}$ algorithm in order to reduce the number of matrix-vector multiplications to two per iteration, while maintaining the benefits of the transformation. Among the different types of CG algorithms for least-squares equations, we choose the LSQR algorithm, which is a stable method based on Lanczos bidiagonalization as detailed in [6]. Then, without having to consider the transformation in (8) explicitly, we simply feed the LSQR algorithm with the matrix-vector multiplications related to the impedance matrix $\bar{Z}$ and its transpose complex conjugate $\bar{Z}^{H}$. Then, LSQR performs the CG solutions of the transformed equation in an implicit and stable way.

\section{RESULTS}

Fig. 3 presents the iterative solutions of a scattering problem with 14871 unknowns. The problem involves a sphere of radius $2 \lambda$ illuminated by a plane wave. Ordinary equations obtained with CFIE and EFIE are solved iteratively by employing various algorithms, including LSQR. We note that the solution of the ordinary equation in (5) using LSQR corresponds to a stable solution of the transformed equation in (8) using the CG algorithm. All the iterative algorithms in Fig. 3 require two matrix-vector multiplications for each iteration except for GMRES, which requires only one matrix-vector multiplication per iterations at the cost of increased memory.

Fig. 3(a) shows that the most efficient solutions of the CFIE formulation are performed by BiCGSTAB and the no-restart
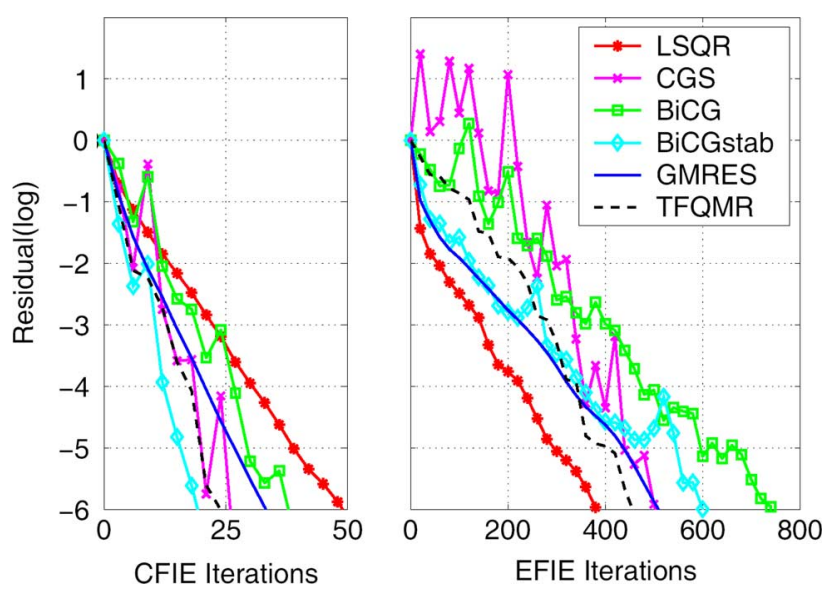

Fig. 3. Iterative solutions of a scattering problem involving a sphere of radius $2 \lambda$ formulated by (a) CFIE and (b) EFIE. Matrix equations with 14871 unknowns are solved by various iterative algorithms.

GMRES. In terms of the processing time, GMRES is better than other iterative algorithms since it requires only 34 matrix-vector multiplications to reduce the residual error to less than $10^{-6}$. However, BiCGSTAB is preferable since it requires only 20 iterations, or 40 matrix-vector multiplications, without using the extra memory needed by GMRES. On the other hand, LSQR is inefficient compared to all other iterative algorithms by requiring 50 iterations, or 100 matrix-vector multiplications, to reduce the error to the same levels. Therefore, LSQR appears to be a bad choice for the CFIE solution of the problem.

In contrast to CFIE, Fig. 3(b) shows that LSQR has significantly different convergence properties for the EFIE solution of the problem; it is not the slowest converging iterative algorithm as in Fig. 3(a). Indeed, it requires 383 iterations, or 766 matrix-vector multiplications, to reduce the error to less than $10^{-6}$ without using the extra memory needed by GMRES, which requires 510 matrix-vector multiplications. In other words, LSQR provides faster convergence compared to BiCG, BiCGSTAB, CGS, and TFQMR, and it is a good alternative to GMRES, which offers a faster convergence at the cost of extra memory. The improved convergence provided by LSQR is due to the favorable properties of the transformation in (8) for EFIE. The same improvement cannot be observed in the CFIE solutions since the transformation in (8) does not improve the convergence of CFIE, as also depicted in Fig. 2.

In Fig. 3, we observe that LSQR provides an improved convergence for EFIE, but it is still inefficient compared to CFIE solutions. In general, for the solutions of problems involving closed surfaces, CFIE is very efficient and negates the necessity of employing LSQR with EFIE. On the other hand, for problems with open surfaces, EFIE is the inevitable choice and the improved convergence provided by LSQR becomes important. As an example, Fig. 4 presents the solution of scattering problems involving a conducting patch depicted in Fig. 1(b), where $d$ has various values from $12 \lambda$ to $30 \lambda$ and the patch is illuminated by a plane wave propagating in the $-z$ direction. Discretizations of the geometry for different frequencies lead to relatively large matrix equations with the number of unknowns ranging from 49200 to 310383 . The scattering problems are solved by using a parallel implementation of MLFMA on an 8-way 


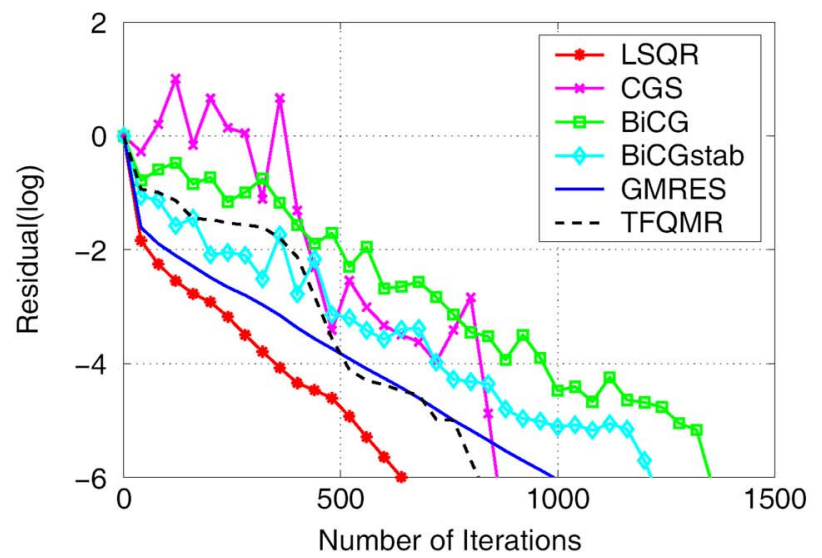

(a)


(b)

Fig. 4. (a) Iterative solutions of a scattering problem involving a $30 \lambda \times 30 \lambda$ patch leading to a matrix equation with 310383 unknowns. (b) Processing time and peak memory per processor with respect to number of unknowns for the solutions of scattering problems involving a patch geometry with various dimensions from $12 \lambda \times 12 \lambda$ to $30 \lambda \times 30 \lambda$. The scattering problems are solved by using a parallel implementation of MLFMA on an 8-way SMP server with dual-core AMD Opteron processors, and only the time and memory required for the iterative solutions are considered.

SMP server with dual-core AMD Opteron processors. Fig. 4(a) presents the iteration counts when $d=30 \lambda$ and the number of unknowns is 310383. In terms of iteration counts, LSQR provides the fastest convergence compared to other iterative algorithms and it requires 642 iterations, or 1284 matrix-vector multiplications, to reduce the error to less than $10^{-6}$. On the other hand, since GMRES requires only one matrix-vector multiplication per iteration, a fair comparison should be based on the processing times of the iterative solutions. In addition, such a comparison is necessary in order to consider different setup times required by the iterative algorithms, which become considerable as the number of unknowns increases. In this manner, Fig. 4(b) presents the processing times for the solutions with respect to the number of unknowns. We ignore the fixed setup time for MLFMA and report only the time required to perform the iterative solutions to reduce the residual error to less than $10^{-6}$. It can be observed that no-restart GMRES provides the fastest solution, especially as the problem size grows. However, as also seen in Fig. 4(b), it requires a peak memory that is significantly larger than the other iterative algorithms. As an example, for the solution of the largest problem in Fig. 4 with 310383 unknowns, GMRES requires $321 \mathrm{MB}$ memory per processor, while the total memory usage (including the memory required by MLFMA) is only $456 \mathrm{MB}$ per processor. Thus, leaving the memory-hungry GMRES aside, among the memory-efficient solutions provided by the other algorithms, LSQR requires the minimum processing time, as depicted in Fig. 4(b). In other words, LSQR performs better than CGS, BiCG, BiCGSTAB, and TFQMR, and it is a strong alternative to no-restart GMRES, which has considerably larger memory requirements.

\section{CONCLUSION}

In this letter, we present improved iterative solutions of EFIE by employing the LSQR algorithm. The accelerated convergence of iterations is especially important for problems with open geometries that cannot be formulated with CFIE. LSQR is a stable way of applying the CG algorithm on the transformed equation obtained by preconditioning the ordinary equation with the transpose complex conjugate of the impedance matrix. Despite the squaring of the condition number due to such a transformation, LSQR improves the rate of convergence of iterative solutions of EFIE, for which it is demonstrated to be as fast as GMRES, and furthermore, it does not need the extra memory build-up required by the no-restart GMRES.

\section{REFERENCES}

[1] D. R. Wilton and J. E. Wheeler III, "Comparison of convergence rates of the conjugate gradient method applied to various integral equation formulations," Progr. Electromagn. Res. PIER 05, pp. 131-158, 1991.

[2] L. Gürel and Ö. Ergül, "Comparisons of FMM implementations employing different formulations and iterative solvers," in Proc. IEEE Antennas Propagat. Soc. Int. Symp., 2003, vol. 1, pp. 19-22.

[3] T. Malas and L. Gürel, "Incomplete LU preconditioning with multilevel fast multipole algorithm for electromagnetic scattering," SIAM J. Sci. Comput., vol. 29, no. 4, pp. 1476-1494, June 2007.

[4] J. Song, C.-C. Lu, and W. C. Chew, "Multilevel fast multipole algorithm for electromagnetic scattering by large complex objects," IEEE Trans. Antennas Propagat., vol. 45, no. 10, pp. 1488-1493, Oct. 1997.

[5] S. Balay, K. Buschelman, V. Eijkhout, W. D. Gropp, D. Kaushik, M. G. Knepley, L. C. McInnes, B. F. Smith, and H. Zhang, PETSc Users Manual, Argonne National Laboratory, 2004.

[6] C. C. Paige and M. A. Saunders, "LSQR: An algorithm for sparse linear equations and sparse least squares," ACM Trans. Math. Software, vol. 8, pp. 43-71, Mar. 1982.

[7] S. M. Rao, D. R. Wilton, and A. W. Glisson, "Electromagnetic scattering by surfaces of arbitrary shape," IEEE Trans. Antennas Propagat., vol. AP-30, no. 3, pp. 409-418, May 1982.

[8] R. J. Adams, "Physical and analytical properties of a stabilized electric field integral equation," IEEE Trans. Antennas Propagat., vol. 52, no. 2, pp. 362-372, Feb. 2004.

[9] R. J. Adams and N. J. Champagne, "A numerical implementation of a modified form of the electric field integral equation," IEEE Trans. Antennas Propagat., vol. 52, no. 9, pp. 2262-2266, Sep. 2004. 